\title{
Pre-treatment effects of walnut kernel (juglans regia) on brain edema, neuronal death and neurological scores in male rat after traumatic brain injury
}

\author{
Javad Mohajer Ansari ${ }^{1}$, Seyed Hassan Eftekhar-Vaghefi ${ }^{1}$, Nader Shahrokhi ${ }^{2}$, Mohsen Basiri ${ }^{3}$, Fatemeh Mehdi Pour ${ }^{3}$, \\ Majid Asadi-Shekaari ${ }^{3 *}$ \\ ${ }^{1}$ Dept of Anatomical sciences, Afzali Pour Medical Sciences, Kerman University of Medical Sciences, Kerman, Iran. \\ ${ }^{2}$ Dept of Physiology, Afzali Pour Medical Sciences, Kerman University of Medical Sciences, Kerman, Iran. \\ ${ }^{3}$ Neuroscience Research Center, Neuropharmocology Institute, Kerman University of Medical Sciences, Kerman, Iran.
}

\begin{tabular}{l} 
ARTICLE INFO \\
\hline Article history: \\
Received on: 04/12/2015 \\
Revised on: 18/02/2016 \\
Accepted on: 27/06/2016 \\
Available online: $29 / 10 / 2016$ \\
\hline Key words: \\
Traumatic brain injury; \\
Walnut kernel; Brain edema; \\
Intracranial pressure.
\end{tabular}

\begin{abstract}
A common pathology in Traumatic brain injury (TBI) is brain edema that develops within hours of impact. It causes increased intracranial pressure (ICP), and nerve damage. It was shown that Walnut kernel (WK) has a large amount of phenolic compounds with beneficial effects on human health due to antioxidant and antiinflammatory properties. The present study was designed to investigate the effect of WK feeding on brain edema, neurological score and neuronal degeneration in male rat after traumatic brain injury. The diffuse TBI was induced in adult male rats using Marmarou's method. Sixty days prior to the injury, WK was added to ordinary food (6\% percent of daily food). Experimental groups are included sham (no TBI and no WK), control (TBI and no WK) and treatment (TBI and WK). Brain edema and neuronal injury were measured $72 \mathrm{~h}$ after TBI. Veterinary Coma Scale (VCS) and ICP were assessed at -1, 4, 24, 48 and $72 \mathrm{~h}$ after TBI. Brain water content and ICP in treatment group decreased as compared to the control. Besides, VCS at 24, 48 and $72 \mathrm{~h}$ after TBI showed a significant increase in treatment group in comparison with control. Based on our data, WK pretreatment may reduce pathological parameters after TBI in male rats.
\end{abstract}

\section{INTRODUCTION}

Traumatic brain injury (TBI) is one of the most important cause of mortality and morbidity among young individuals worldwide.TBI produces an inflammatory reaction that is usually accompanied by intense cell death in different areas of the brain (Saatman et al., 2006). The cytotoxic events can directly affect patient outcome after TBI, which can be further exacerbated by uncontrolled intracranial pressure (ICP) (Jiang et al., 2002) caused by an increase in brain water content (Griebenow et al., 2007). Uncontrolled ICP can produce greater

\footnotetext{
* Corresponding Author
}

Asadi-Shekaari Majid, Ebn Sina Avenue, Neuroscience Research Center, Neuropharmacology institute, Kerman University of Medical Sciences, Kerman, Iran.E-mail: Majidasadi @ kmu.ac.ir; Tel: +983432264251 secondary damage through ischemia (Stiefel et al., 2005) and an increase in mortality caused by hernia of the brain (Lang and Chesnut, 1995). Up to now, TBI treatment methods have concentrated on the reduction of ICP and post oxidative stress, but an effective pharmacological treatment remains to be found.

Walnut species are the main source of nuts in mild climate zones around the world. In Iran, Juglans regia L. (Juglandaceae) is not just an agricultural product: its leaves, fruit, stems and flowers are all used for different medicinal purposes. Walnut kernels (WK) have high concentrations of phenolic compounds, which have positive effects on human health because of their anticonvulsive, neuroprotective, antioxidant and anti-atherogenic effects $(\mathrm{Li}$ et al., 2007, Asadi-Shekaari et al., 2012). In addition, walnut kernels are a nutrient-rich food, containing plentiful phospholipids, proteins, unsaturated fatty acids and tocopherols (Labukas et al., 2010). 
Currently, there is no F.D.A approved treatment for complication reduction after TBI (McAllister et al., 2001). Hence, this study was designed to investigate the effect of walnut kernel feeding on brain edema, neurological score and neuronal degeneration in male rat after traumatic brain injury.

\section{MATERIALS AND METHODS}

This study was conducted in accordance with the guidelines for the animal experimental protocols of Kerman University of Medical Sciences. The protocol was approved by the ethics committee (EC/KNRC/91-14) of this university, in accordance with the internationally accepted principles for laboratory animal use and care, as found in the US guidelines (NIH publication \#85-23, revised in 1985) or European Community guidelines (EEC Directive of 1986; 86/609/EEC). Adult male Albino Wistar rats (weighing 180-200 g) were housed in an air-conditioned room in Afzali Pour Medical Faculty, Kerman University of Medical Sciences, at 22-24 ${ }^{\circ} \mathrm{C}$, with a $12 \mathrm{~h}$ light : $12 \mathrm{~h}$ dark cycle and free access to food and water. Animals $(n=42)$ were divided into three groups of sham (no TBI and no WK), control (TBI and no WK) and treatment (TBI and WK, 6\% percent of daily food for 2 months).

The number of animals in each group was 14 . The groups were divided into two subgroups $(n=7)$ for measuring brain water content and neurologic score (subgroup 1), and neuronal injury (subgroup 2).

\section{Induction of TBI}

The TBI method of the diffuse type induced by the Marmarou's method (Marmarou et al., 1994), using a TBI induction device made by the Dept. of Physiology, Afzali Pour Medical Faculty, Kerman University of Medical Sciences. The protocol was as follows: a $250 \mathrm{~g}$ weight was dropped from a $2 \mathrm{~m}$ height on the head of the anesthetized (chloral hydrate, $380 \mathrm{mg} / \mathrm{kg}$ ) animal rat while a metal disc (stainless steel, $10 \mathrm{~mm}$ in diameter, $3 \mathrm{~mm}$ thick) was attached to the animal's skull. Control (shamoperated) animals were anesthetized and had the steel disc attached to the skull, but they did not receive the weight drop. After TBI induction, the rats were immediately connected to a respiratory pump (TSA animal respiratory compact, Bad Homburg, Germany). After spontaneous breathing had been restored, the intratracheal tube was removed and, following recovery, the rats were placed in individual cages (Shahrokhi et al., 2010).

\section{Determination of Brain Edema}

Edema was measured by assessing water content in the brains. The weight of wet tissue was assessed first and then incubated in $70^{\circ} \mathrm{C}$ in an incubator (Memmert, Germany) for 72 hours to evaporate the tissue water and dry, the brain was weighed again and water content was determined by using the formula listed below:

Brain water content $\%=[($ wet weight-dry weight $) /$ wet weight $] \times 100$

\section{Evaluation of Neurological Outcomes}

According to veterinary coma scale (VCS), in the range of 3-15 that was the sum of 3 parts: motor function (score range 18 ), respiration (score range 1-3), and eye function (score range 14). Based on VCS criteria, higher scores show better neurological outcomes, and lower scores show worse neurological outcomes. In the current study, the outcomes were measured 1 hour before trauma induction and measurements were continued at , 4, 24, 48 and 72 hours after TBI (Stahel et al., 2000).

\section{FluoroJade (FJ) staining}

At $72 \mathrm{~h}$ after TBI, animals underwent intra-cardiac perfusion with saline $(0.9 \% \mathrm{NaCl}$ in distilled water) and $4 \%$ paraformaldehyde, subsequent to which the brain was extracted. The right hemisphere was processed for FJ staining and the left one for Nissl staining. FJ histochemistry was carried out as originally described (Schmued et al., 1997). In brief, brain coronal sections containing the sensorimotor cortex were washed in $80 \%$ ethanol with $1 \% \mathrm{NaOH}, 70 \%$ ethanol in distilled water. Then, tissue sections were washed in $0.06 \% \mathrm{KMnO} 4$ for $15 \mathrm{~min}$ and washed again in distilled water. The FJ solution ( $1 \mathrm{ml})$ was diluted in $99 \mathrm{ml} 0.1 \%$ acetic acid and the tissue was incubated for $15 \mathrm{~min}$. Sections were then rinsed in distilled water, dehydrated, air dried, and mounted on gelatin coated slides. Then nuclei were stained by 4'-6-diamidino-2-phenylindole (DAPI) to show total cells.

\section{Nissl staining}

For quantative analysis, three days after TBI, the brains (left hemisphere) were processed consistent with standard histological methods. Paraffinized brains were cut into $6 \mu \mathrm{m}$ sections on a rotary microtome and the sections were stained with Cresyl fast violet (Nissl method). Neuronal injury was then determined for each animal as the rate of the number of degenerated pyramidal neurons to that of both surviving and degenerated in three distinct fields of the cortex in sections (AsadiShekaari et al., 2012).

\section{Statistical Analysis}

Statistical analysis was carried out with SPSS for Windows. All the data represented as mean \pm SEM. Differences among multiple groups were assessed using ANOVA. Tukey's test was used for the ANOVA post-hoc analysis. The criterion for statistical significance was sign at $p<0.05$.

\section{RESULTS}

\section{Determination of water content}

The water content of the brain in the different groups, at $72 \mathrm{~h}$ post TBI is shown in Fig.1.This figure demonstrates that the brain water content in control group is significantly more than in sham group $(\mathrm{p}<0.05)$, on the other hand the brain water content in treatment group was significantly less than that of control groups $(\mathrm{p}<0.05)$. 


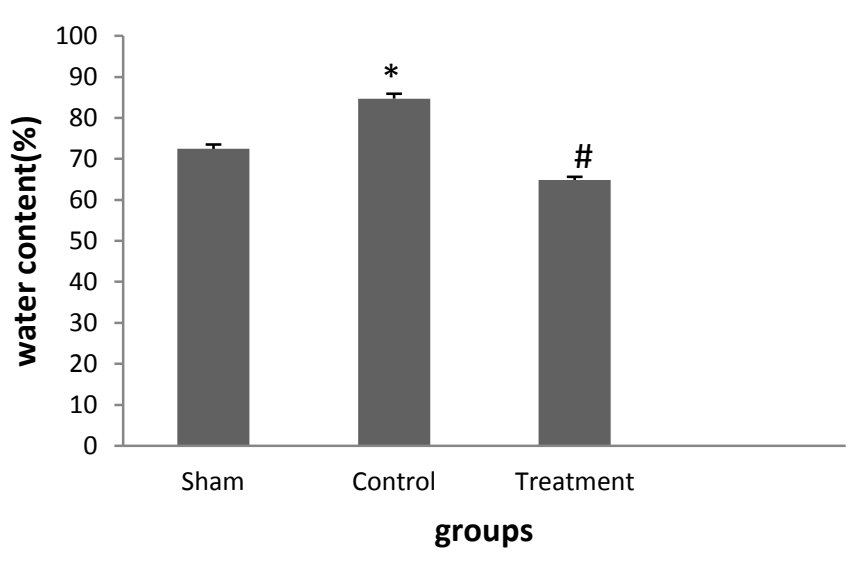

Fig. 1: The effect of WK-pretreatment on brain water content $72 \mathrm{~h}$ after inducing TBI in male rats. Experimental groups are included sham (no TBI and no WK), control (TBI and no WK) and treatment (TBI and WK). Data are presented as mean \pm SEM, $n=7 /$ groups. *: Significant difference between control and sham groups $(\mathrm{p}<0.05)$. \#: Significant difference compared to sham group $(\mathrm{p}<0.05)$.

\section{Neurological score}

Experimental time course including 4, 24, 48, and 72 hours has shown significance difference between treated animal groups comparing to control and/or sham groups $\mathrm{P}<0.05$ or $\mathrm{P}<$ 0.001 . However, no significance differences were seen following 1 hour in our experimental groups.

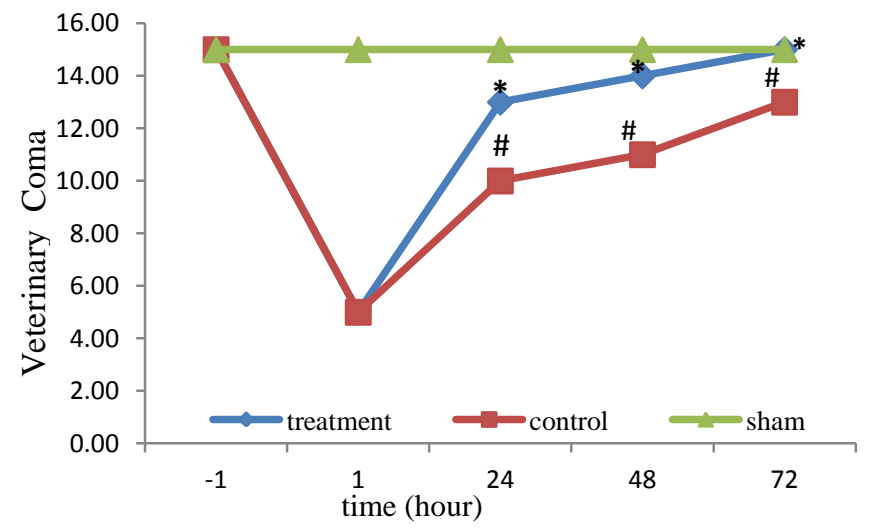

Fig. 2: The effect of WK-treatment on neurological scores after inducing TBI in male rats. Experimental groups are included sham (no TBI and no WK), control (TBI and no WK) and treatment (TBI and WK). ***: Significant difference in compared to sham group $(\mathrm{p}<0.001)$. Neurological scores increased in TBI groups that pre-treated with WK group compared to control group \# $\mathrm{P}<0.05$. Data are presented as mean $\pm \mathrm{SEM}, \mathrm{n}=7$ /group.

\section{Neuronal degeneration}

Qualitative Neuronal degeneration after TBI was assessed by FluoroJade (FJ) staining at $72 \mathrm{~h}$ post trauma. Compared to sham (Fig. 3A), intense FJ-labeled neuronal perikarya were detected in the cortex (Fig. 3B). While, in all regions, FJ labeled cells were significantly reduced as a result of pre-treatment with WK (Fig. 3C). Compared to the control, the injured cortex in animals pre-treated with WK demonstrated reduction in neuronal injury.

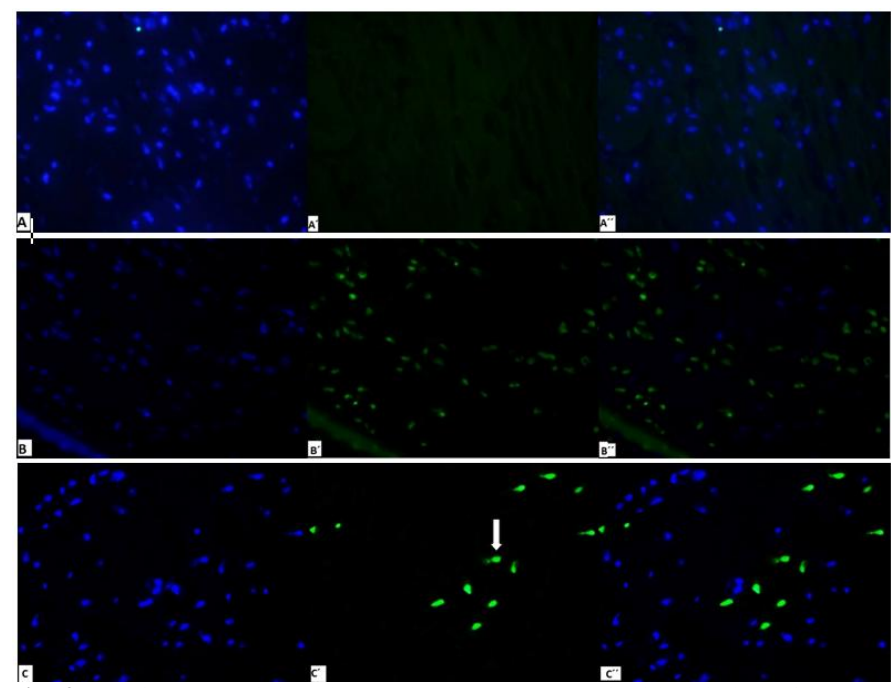

Fig. 3: Sections containing the cerebral cortex were double-labeled with DAPI (blue nuclei show total cells) (A, B, C) and Fluoro-Jade (A',B',C'), and their merged (A",B",C") to show degeneration of neurons. Fluoro-Jade staining of the sections demonstrates the extensive neuronal damage that has occurred in control group (TBI and no WK) (B) comparing to sham group (no TBI and no WK) (A). Pre-treatment with WK decreased neurodegeneration in cerebral cortex as observed in the figure $(\mathrm{C}, \mathrm{D})$. White arrow shows degenerated neuron. Magnification: $\times 100$.

\section{Neuronal counting}

Our data showed that TBI induced severe neuronal degenration in the brain cortex of control group (54.13\%). Pretreatment with WK significantly diminished degeneration in treatment group $(22.37 \%)$. Neuronal degenerated was in sham group was (7.48\%) (Fig. 4).

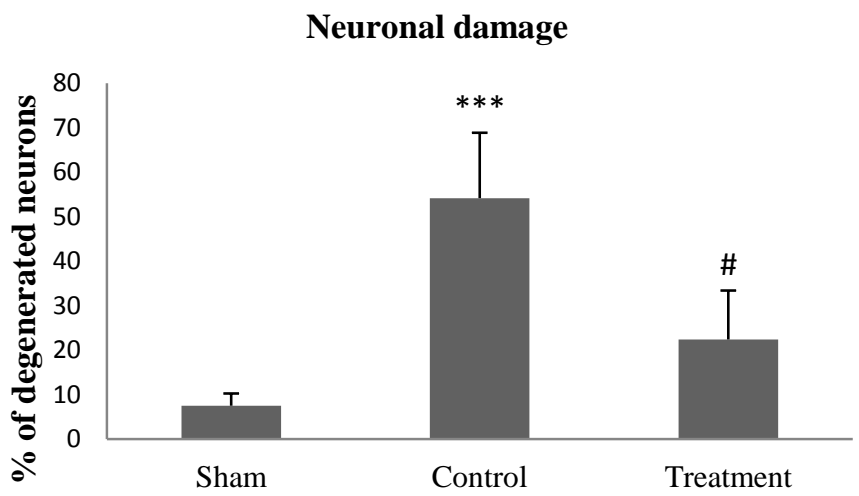

Fig. 4: Neuronal damage in cerebral cortex $72 \mathrm{hr}$ after TBI was analyzed using Nissl's staining. Experimental groups are included sham (no TBI and no WK), control (TBI and no WK) and treatment (TBI and WK). Results were expressed as Mean \pm SEM. A statistical difference was determined by a value of 0.05 .

\section{DISCUSSION}

In the current study, we investigated the effect of WK diet in the development of brain edema, neurological scores, and neuronal degeneration after TBI in male rats. The main finding was acquired in this study; WK pre-treatment reduced brain edema, improved neurological scores and prevented increased neuronal degeneration post-TBI, in male rats. 
The results of experimental studies have revealed that blood-brain barrier (BBB) breakdown and increase of endothelium permeability after brain trauma can cause brain edema and eventually ICP increase (Shlosberg et al., 2010). Brain edema resulted in cell injury after TBI (Papadopoulos and Verkman, 2007). It has shown that edema causes a rise in ICP, which contributes significantly to neuronal cell injury (Griesbach et al., 2009), and also enhances the mortality and morbidity rates in TBI patients (Sarabia et al., 1988). It seems that oxidative stress has a main role in post-TBI neuronal injury (Marmarou, 2007). Accumulation of oxygen-derived free radicals in vessels has a key role in the molecular cascade involved in BBB breakdown (del Zoppo, 2006). Moreover, inflammation originated from TBI resulted in secondary vascular damage and intense neuronal degeneration in the brain (Shlosberg et al., 2010; Saatman et al., 2006).

The results of our study demonstrated that TBI causes severe neuronal degeneration in the brain cortex, while, pretreatment with WK ameliorates the induced injury. The neuroprotective effect of KW was confirmed by Asadi-Shekaari et al. They stated that the positive effects of the WK are dependent upon its anti-inflammatory and antioxidant properties (AsadiShekaari et al., 2014).

Walnut kernels contain essential fatty acids such as alpha-linolenic acid (ALA), linoleic acid (LA) and oleic acid. In addition to high fatty acid contents, the walnut has some other beneficial constituents such as vitamin E, melatonin and numerous antioxidant polyphenolics. Different polyphenols of WK include tannins, ellagic acid, gallic acid and glansrins A, B and C (Ito et al., 2007).

According to a recent study, walnut extract reduced the production of Tumor Necrosis Factor- $\alpha$ (TNF- $\alpha$ ) in BV-2 microglial cells activated by lipopolysaccharide (LPS) (Liu et al., 2012). Walnut extract and its component ellagic acid also showed anti-inflammatory activity in human aorta endothelial cells and osteoblastic activity in the cell line KS483 (Papoutsi et al., 2008). In addition, a more recent study showed that fatty acids have a role in reduction of inflammation response in peritoneal macrophages (Montserrat-de la Paz et al., 2014). In our study, the reduction of neuronal degeneration in pre-treatment group may be due to antiinflammatory constituents of WK.

Neurologic scores of WK pre-treated group at different post TBI h were higher compared to the control group. It has been reported that low neurologic scores are associated with vasoconstriction and cerebral hypoperfusion (Shahlaie et al., 2009). Nuts including walnuts contain large amounts of Larginine, the precursor amino acid of the endogenous vasodilator nitric oxide (NO)(Cooke et al., 1993). Moreover, one of the beneficial components of $\mathrm{WK}$ is melatonin (Al-Qarawi et al., 2003) that may be involved in the protective effect of WK in treated group. Recently, Dehghan et al.,confirmed that melatonin can increase neurologic scores after TBI in male rats (Dehghan et al., 2013). Although the precise mechanism of this protective effect is not yet known, but high L-arginine content and melatonin could be an explanation for the observed effect.

According to our data, WK pre-treatment for 60 days prior to TBI is effective in decreasing brain edema, and neuronal degeneration, as well as in improving neurological scores. These positive properties may be due to its potent antioxidant and antiinflammatory effects.

\section{CONCLUSION}

WK diet might decrease brain edema, and neuronal degeneration, plus improving neurological scores in TBI group. This may suggest that WK consumption has beneficial effects in reducing the injury caused by TBI, nevertheless, the precise mechanism of anti-inflammatory and antioxidative properties of WK require further studies.

\section{ACKNOWLEDGMENTS}

The data presented in this article are from a Master thesis (Javad Mohajer Ansari) performed in the neuroscience Research Center of KUMS.

Source of financial Support: This study was supported by a grant from Neuroscience Research Center of Kerman University of Medical Sciences (KUMS).

Conflict Of Interest: The authors declare no conflict of interest.

\section{REFERENCES}

Saatman KE, Feeko KJ, Pape RL, Raghupathi R. Differential behavioral and histopathological responses to graded cortical impact injury in mice. J Neurotrauma, 2006; 23:1241-1253.

Jiang Jy, Gao GY, Li WP, Yu MK, Zhu C. Early indicators of prognosis in 846 cases of severe traumatic brain injury. J Neurotrauma, 2002; 19:869-874.

Griebenow M, Casalis P, Woiciechowsky C, Majetschak M, Thomale UW. Ubiquitin reduces contusion volume after controlled cortical impact injury in rats. J Neurotrauma, 2007; 24: 1529-1535.

Stiefel MF, Tomita Y, Marmarou A. Secondary ischemia impairing the restoration of ion homeostasis following traumatic brain injury. J Neurosurg, 2005; 103:707-14.

Lang EW, Chesnut RM. Intracranial pressure and cerebral perfusion pressure in severe head injury. New Horiz, 1995;3:4009.

Li L, Tsao R, Yang R, Kramer JK, Hernandez M. Fatty acid profiles, tocopherol contents, and antioxidant activities of heartnut (Juglans ailanthifolia Var. cordiformis) and Persian walnut (Juglans regia L.). J Agric Food Chem, 2007; 55:1164-9.

Asadi-Shekaari M, Kalantaripour TP, Nejad FA, Namazian E, Eslami A. The anticonvulsant and neuroprotective effects of walnuts on the neurons of rat brain cortex. Avicenna J Med Biotechnol, 2012; 4:155158.

Labukas JP, Drake TJ, Ferguson GS. Compatibility of omegafunctionality in the electrochemically directed self-assembly of monolayers on gold from alkyl thiosulfates. Langmuir, 2010; 26:497-505.

Mcallister TW, Sparling MB, Flashman LA, Guerin SJ, Mamourian AC, Saykin AJ. Differential working memory load effects after mild traumatic brain injury. Neuroimage, 2014;14:1004-1012.

Shahrokhi N, Khaksari M, Soltani Z, Mahmoodi M, Nakhaee N. Effect of sex steroid hormones on brain edema, intracranial pressure, and 
neurologic outcomes after traumatic brain injury. Can J Physiol Pharmacol, 2010;88:414-21.

Stahel PF, Shohami E, Younis FM, KariyaK, Otto VI, Lenzlinger PM, Grosjean MB, Eugster HP, Trentz O, Kossmann T, Morganti-Kossmann MC. Experimental closed head injury: analysis of neurological outcome, blood-brain barrier dysfunction, intracranial neutrophil infiltration, and neuronal cell death in mice deficient in genes for pro-inflammatory cytokines. J Cereb Blood Flow Metab, 2000;20:36980 .

Marmarou A, Foda MA, Van Den Brink W, Campbell J, Kita H, Demetriadou K. A new model of diffuse brain injury in rats. Part I: Pathophysiology and biomechanics. J Neurosurg, 1994;80:291-300.

Schmued LC, Albertson C, Slikker W. Fluoro-Jade: a novel fluorochrome for the sensitive and reliable histochemical localization of neuronal degeneration. Brain Res, 1997;751:37-46.

PapadopoulosMC, Verkman AS. Aquaporin-4 and brain edema. Pediatr Nephrol, 2007;22:778-84.

Griesbach GS, Hovda DA, Gomez-Pinilla F. Exercise-induced improvement in cognitive performance after traumatic brain injury in rats is dependent on BDNF activation. Brain Res, 2009;1288:105-15.

Sarabia R, Lobato RD, Rivas JJ, Cordobes F, Rubio J, Cabrera A, Gomez P, Munoz MJ, Madera A. Cerebral hemisphere swelling in severe head injury patients. Acta Neurochir Suppl (Wien), 1988;42:40-6.

Marmarou A. A review of progress in understanding the pathophysiology and treatment of brain edema. Neurosurg Focus, 2007;22:1-10.

Del Zoppo GJ. Stroke and neurovascular protection. N Engl J Med, . 2006;354:553-5.

Shlosberg D, Benifla M, Kaufer D, Friedman A. Blood-brain barrier breakdown as a therapeutic target in traumatic brain injury. Nat Rev Neurol, 2010;6:393-403.

Asadi-Shekaari M, Basiri M, Babaee A. The protective effects of walnuts (Juglans regia) on neuronal death and astrocyte reactivity following middle cerebral artery occlusion in male rats. Scientific Journal of Kurdistan University of Medical Sciences, 2014;19:100-108.

Ito H, Okuda T, Fukuda T, Hatano T, Yoshida T. Two novel dicarboxylic Acid derivatives and a new dimeric hydrolyzable tannin from walnuts. J Agric Food Chem, 2007; 55: 672-9.
Liu T, Li J, Liu Y, Xiao N, Suo H, Xie K, Yang C, Wu C. Short-chain fatty acids suppress lipopolysaccharide-induced production of nitric oxide and proinflammatory cytokines through inhibition of NFkappaB pathway in RAW264.7 cells. Inflammation, 2012;35:1676-84.

Papoutsi Z, Kassi E, Chinou I, Halabalaki M, Skaltsounis LA, Moutsatsou P. Walnut extract (Juglans regia L.) and its component ellagic acid exhibit anti-inflammatory activity in human aorta endothelial cells and osteoblastic activity in the cell line KS483. Br J Nutr, 2008;99:715-22.

Montserrat-De La Paz S, Garcia-Gimenez MD, Angel-Martin M, Perez-Camino MC, Fernandez Arche A. Long-chain fatty alcohols from evening primrose oil inhibit the inflammatory response in murine peritoneal macrophages. J Ethnopharmacol, 2014;151:131-6.

Shahlaie K, Boggan JE, Latchaw RE, Ji C, Muizelaar JP. Posttraumatic vasospasm detected by continuous brain tissue oxygen monitoring: treatment with intraarterial verapamil and balloon angioplasty. Neurocrit Care, . 2009;10:61-9.

Cooke JP, Tsao P, Singer A, Wang BY, Kosek J, Drexler H. Anti-atherogenic effect of nuts: is the answer NO? Arch Intern Med, 1993; 153,896:899, 902.

Al-Qarawi AA, Ali BH, Al-Mougy SA, Mousa HM. Gastrointestinal transit in mice treated with various extracts of date (Phoenix dactylifera L.). Food Chem Toxicol, 2003;41:37-9.

Dehghan F, Khaksari Hadad M, Asadikram G, Najafipour H, Shahrokhi N. Effect of melatonin on intracranial pressure and brain edema following traumatic brain injury: role of oxidative stresses. Arch Med Res, 2013;44:251-8.

\section{How to cite this article:}

Ansari JM, Eftekhar-Vaghefi SH, Shahrokhi N, Basiri M, Pour FM, Asadi-shekaari M. Pre-treatment effects of walnut kernel (juglans regia) on brain edema, neuronal death and neurological scores in male rat after traumatic brain injury. J App Pharm Sci, 2016; 6 (10): 102-106. 